\title{
Comparing Multilateral and Bilateral Exchange Models for Content Distribution
}

\author{
Christina Aperjis \\ Stanford University
}

\author{
Michael J. Freedman \\ Princeton University
}

\author{
Ramesh Johari \\ Stanford University
}

Users of peer-to-peer systems are often incentivized to contribute their upload capacity in a bilateral manner: downloading is possible in return for uploading to the same peer (e.g., BitTorrent). An alternative is to use multilateral exchange to match user demand for content to available supply at other peers in the system. Multilateral exchange can be enabled through prices and a virtual currency. Monetary incentives have been previously proposed to incentivize uploading in $\mathrm{P} 2 \mathrm{P}$ systems [1], [2], [3], [4]. We provide a formal comparison of P2P system designs based on bilateral exchange with those that enable multilateral exchange via a price-based market mechanism to match supply and demand. This work surveys and generalizes [5] and [6].

We start with a fundamental abstraction of content exchange in systems with bilateral barter: exchange ratios. The exchange ratio from one peer to another gives the download rate received per unit upload rate. We show that exchange ratios can be used to model filesharing systems such as BitTorrent and variants like BitTyrant. Moreover, exchange ratios are a useful formal tool that directly allows us to compare bilateral P2P systems with price-based multilateral $\mathrm{P} 2 \mathrm{P}$ systems. We compare the allocations that arise at equilibria of bilateral P2P systems using exchange ratios, with those that arise at equilibria of price-based multilateral P2P systems.

We consider a set of peers that shares a set of files $F$. Peer $i$ has a subset of the files $F_{i} \subseteq F$, and is interested in downloading files in $T_{i} \subseteq F-F_{i}$. We use $r_{i j f}$ to denote the rate at which user $i$ uploads file $f$ to user $j$. We then let $d_{i f}=\sum_{j} r_{j i f}$ be the total rate at which user $i$ downloads file $f$. We measure the desirability of a download vector to peer $i$ by a utility function $V_{i}\left(\boldsymbol{d}_{i}\right)$ that is nondecreasing in every $d_{i f}$ for $f \in T_{i}$. Peer $i$ incurs a cost $c_{i}\left(y_{i}\right)$ for uploading at rate $y_{i}$.

Given a vector of exchange ratios $\gamma$, where $\gamma_{i j}$ is the ratio at which peer $i$ can exchange with peer $j$, peer $i$ solves the following optimization problem.

$$
\begin{array}{ll}
\operatorname{maximize} & V_{i}\left(\boldsymbol{d}_{i}\right)-c_{i}\left(y_{i}\right) \\
\text { subject to } & d_{i f}=\sum_{j} r_{j i f}, \forall f \\
& r_{k j f}=0, \text { if } f \notin F_{k} \\
& \sum_{j, f} r_{i j f} \leq y_{i} \\
& \sum_{f} r_{j i f}=\gamma_{i j} \sum_{f} r_{i j f}, \forall j \\
& \boldsymbol{r} \geq 0
\end{array}
$$

Note that we allow peers to bilaterally exchange content over multiple files; this is a more general design, even though this is not typically supported by swarming systems like BitTorrent. This more general design makes it possible, even in bilateral exchange, to explicitly identify the relative demand for files and reward peers that share more popular content.

By contrast, in a multilateral price-based exchange, the system maintains one price per peer, and peers optimize with respect to these prices. Denote the price of a peer $i$ by $p_{i}$. Given a price vector $\boldsymbol{p}$, peer $i$ solves the following optimization problem.

$$
\begin{array}{ll}
\operatorname{maximize} & V_{i}\left(\boldsymbol{d}_{i}\right)-c_{i}\left(y_{i}\right) \\
\text { subject to } & d_{i f}=\sum_{j} r_{j i f}, \forall f \\
& r_{k j f}=0, \text { if } f \notin F_{k} \\
& \sum_{j} p_{j} \sum_{f} r_{j i f}=p_{i} y_{i} \\
& \boldsymbol{r} \geq 0
\end{array}
$$

Note that the first two constraints (giving download rates and ensuring peers only upload files they possess) are identical to the bilateral peer optimization. While the bilateral exchange requires peer $i$ to download only from those peers to whom he uploads, no such constraint is imposed on multilateral exchanges: peer $i$ accrues capital for uploading, and he can spend this capital however he wishes for downloading.

For bilateral (resp., multilateral) exchange, an equilibrium is a combination of a rate allocation vector and an exchange ratio vector (resp., price vector) such that all peers have solved their corresponding optimization problems. In this case, the exchange ratios (resp., prices) have exactly aligned supply and demand: for any $i, j, f$, the transfer rate $r_{i j f}$ is simultaneously an optimal choice for both the uploader $i$ and downloader $j$.

\section{AXIOMATIC COMPARISON}

We perform an axiomatic comparison of the rate allocations that arise as bilateral and multilateral equilibria. We first show that multilateral equilibria satisfy some desirable properties that bilateral equilibria do not, and then identify the "gap" between the two types of exchange.

A multilateral equilibrium allocation is Pareto efficient, i.e., there is no way to increase the utility of some peer without decreasing the utility of some other peer. We illustrate by examples that a bilateral equilibrium allocation may not be (Pareto) efficient. This inefficiency arises partly because in 
some settings a subset of peers may not be able to exchange. However, this is not the only reason for inefficiencies: we show that a bilateral equilibrium allocation may be inefficient even if all peers are able to exchange.

We next compare bilateral and multilateral equilibria in terms of whether they are robust to collusive deviations using the notion of the core. An allocation has the core property with respect to given exchange ratios if no coalition of peers can strictly improve the utility of all its members by bartering with peers outside the coalition, subject to the given exchange ratios. Inside the coalition, peers do not need to follow the exchange ratios, and they may allocate rates in any way.

We give an example of a bilateral equilibrium that does not satisfy the core property, thus demonstrating that bilateral equilibria are not in general robust to collusive deviations. On the other hand, we show that the core property is satisfied by any multilateral equilibrium. A multilateral equilibrium allocation cannot be blocked by any coalition of peers, because essentially all peers have already optimized with respect to the same prices. Finally, we show that robustness to collusive deviations is exactly the "gap" between bilateral and multilateral exchange. The following proposition is one of our main results.

Proposition 1 Let $\boldsymbol{r}$ be a bilateral equilibrium allocation, and $\gamma$ the corresponding exchange ratios. There exists a price vector $\boldsymbol{p}$ such that $\boldsymbol{r}$ and $\boldsymbol{p}$ constitute a multilateral equilibrium if and only if $\boldsymbol{r}$ is in the core with respect to $\gamma$.

\section{QUANTITATIVE COMPARISON}

After having axiomatically compared bilateral and multilateral equilibria, we compare the two types of exchange through the expected proportion of peers that can trade. Bilateral exchange may be particularly restrictive because a pair of peers can exchange only if each has a file that the other wants. On the other hand, allowing multilateral exchange significantly increases the number of possible exchanges, and potentially increases the number of peers that can trade.

We assume that each peer has one file and wants one file, and that these files are drawn from a known distribution independently and identically for each peer. Once randomness is realized, peer $i$ is able to trade under bilateral exchange if and only if there exists a peer $j$ with which "double coincidence of wants" is satisfied, i.e., peer $j$ wants the file $i$ has and has the file $i$ wants. Peer $i$ is able to trade multilaterally if and only if there exists a cycle of peers starting (and ending) at $i$ such that "double coincidence of wants" is satisfied for each pair of consecutive peers. Clearly, if peer $i$ can trade bilaterally then it can also trade multilaterally.

We study the performance of bilateral and multilateral exchange in large systems. Let $N$ be the number of peers and $K$ the number of files in the system. For a given distribution, let $g_{B E}(K, N)$ and $g_{M E}(K, N)$ be the expected percentages of peers that do not trade at bilateral and multilateral exchange respectively. Since the number of peers that can not trade bilaterally is always greater or equal to the number of peers that can not trade multilaterally, we have that $g_{B E}(K, N)-g_{M E}(K, N) \geq 0$. The following proposition identifies a setting for which $g_{B E}(K, N)-g_{M E}(K, N)$ is significant.

Proposition 2 Assume files are chosen according to the uniform distribution.

1) If $K \geq \sqrt{N}$, then $g_{B E}(K, N) \rightarrow c>0$ as $N \rightarrow \infty$.

2) If $N^{1-\varepsilon} \geq K$ for some $\varepsilon>0$, then $g_{M E}(K, N) \rightarrow 0$ as $N \rightarrow \infty$.

We conclude that if $N^{1-\varepsilon} \geq K \geq \sqrt{N}$ and files are chosen uniformly, then multilateral exchange performs significantly better than bilateral exchange. However, there exist distributions for which bilateral exchange performs well for any scaling of $K$ and $N$ when the system is large.

Proposition 3 If files are chosen according to a Zipf distribution with $s>1$, then $g_{B E}(K, N) \rightarrow 0$ as $N \rightarrow \infty$ for any scaling of $K$ with $N$.

To summarize, even though bilateral equilibria do not always satisfy some desirable properties that multilateral equilibria have, bilateral exchange performs very well in expectation for certain file popularity distributions.

\section{REFERENCES}

[1] M. Gupta, P. Judge, and M. Ammar, "A reputation system for peer-to-peer networks," in NOSSDAV, 2003.

[2] V. Vishnumurthy, S. Chandrakumar, and E. G. Sirer, "KARMA: A secure economic framework for P2P resource sharing," in WEIS, 2003.

[3] M. Sirivianos, J. H. Park, X. Yang, and S. Jarecki, "Dandelion: Cooperative content distribution with robust incentives," in USENIX Technical, 2007.

[4] C. Aperjis and R. Johari, "A peer-to-peer system as an exchange economy," in GameNets, 2006.

[5] C. Aperjis, M. J. Freedman, and R. Johari, "A comparison of bilateral and multilateral exchanges for peer-assisted content distribution," in NetCoop, 2008.

[6] $\frac{}{2008}$, "Peer-assisted content distribution with prices," in ACM CoNEXT, 